\title{
Estudo Histomorfométrico de Anastomoses Primárias de Cólon em Coelhos, com e sem Preparo Intestinal
}

\author{
Histomorfometric Analysis of Colonic Anastomosis in Rabbits \\ With and Without Intestinal Preparation
}

\author{
JUVENAL DA ROCHATORRES NETO ${ }^{1}$, RICARDO FAKHOURI ${ }^{2}$, MARCEL VINÍCIUS DE \\ AGUIAR MENEZES ${ }^{3}$, JOSEANE SILVASANTOS ${ }^{4}$, ANA CAROLINALISBÔAPRUDENTE ${ }^{5}$, \\ JOÃOTIAGO SILVAMONTEIRO ${ }^{6}$, VERALÚCIACORRÊAFEITOSA $^{7}$
}

\begin{abstract}
1. Professor Adjunto do Departamento de Medicina da Universidade Federal de Sergipe. Coloproctologista do Hospital Universitário da Universidade Federal de Sergipe; ${ }^{2}$ Professor Adjunto do Departamento de Medicina da Universidade Federal de Sergipe. Patologista do Hospital Universitário da Universidade Federal de Sergipe; ${ }^{3}$ Médico-residente em Cirurgia Geral do Hospital Santo Antônio e obras Sociais Irmã Dulce. Salvador-Bahia; ${ }^{4}$ Acadêmica de Medicina da Universidade Federal de Sergipe. Aracaju - Sergipe; ${ }^{5}$ Médica-residente em Coloproctologia do Hospital Universitário da Universidade Federal de Sergipe; ${ }^{6}$ Acadêmico de Medicina da Universidade Federal de Sergipe. Aracaju - Sergipe; ${ }^{7}$ Professora Adjunta do Departamento de Morfologia da Universidade Federal de Sergipe. Bióloga do Departamento de Morfologia da Universidade Federal de Sergipe.
\end{abstract}

TORRES NETO JR, FAKHOURI R, MENEZES MVA, SANTOS JS, PRUDENTE ACL, MONTEIRO JTS E FEITOSA VLC. Estudo Histomorfométrico de Anastomoses Primárias de Cólon em Coelhos, Com e Sem Preparo Intestinal. Rev bras Coloproct, 2007;27(4): 384-390.

RESUMO: O preparo intestinal é muito utilizado em cirurgias do cólon. A LIATO (lavagem intestinal anterógrada trans-operatória) promove limpeza do cólon, conferindo incremento de tempo ao ato cirúrgico e maior risco de infecção pela maior manipulação do conteúdo intestinal .Este estudo compara confecção de anastomoses colônicas com e sem preparo intestinal, pela análise histomorfométrica. Foram submetidos à cirurgia 30 coelhos divididos em 2 grupos: grupo 1, controle e grupo 2, que submeteu-se a LIATO, e comparados os seus resultados. A presença de infiltrado inflamatório agudo teve média discretamente maior nas anastomoses do grupo 2. Infiltrado inflamatório crônico obteve média de 1,9 nas anastomoses do grupo 2 e de 2,1 nas sem preparo. Necrose esteve presente em $15,7 \%$ dos casos onde se realizou LIATO contra 13,5\% no grupo sem preparo. Calcificações foram encontradas em $43 \%$ das anastomoses com preparo e em $30 \%$ das sem preparo. Observou-se maior quantidade de colágeno nas anastomoses feitas com a lavagem intestinal. O padrão entrelaçado das fibras colágenas foi observado em $86 \%$ das anastomoses do grupo 2 e $70 \%$ no 1. Estudo estatístico foi realizado com programa Prism ${ }^{\circledR} 4$ para $p<0,05$. Neste estudo que a LIATO apresenta melhor padrão de cruzamento e concentração de fibras colágenas, resultando possivelmente em melhor cicatrização.

Descritores: Anastomose, intestinal, LIATO, cicatrização, colágeno.

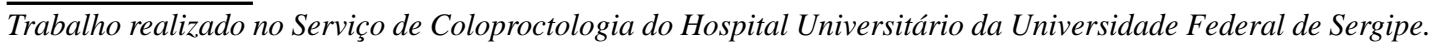

Recebido em 14/08/2007

Aceito para publicação em 04/10/2007 


\section{INTRODUÇÃO}

O preparo intestinal é ainda considerado uma etapa essencial nas cirurgias colo-retais com ressecção e anastomose.

Em cirurgia de urgência, pode optar-se por lavagem intestinal anterógrada trans-operatória (LIATO) antes de realizar as anastomoses colônicas, no entanto, envolve aumento do tempo operatório, do risco de contaminação da cavidade abdominal com fezes durante a lavagem e do risco de infecção ${ }^{(1,2,3,4)}$.

Trabalhos mostram bons resultados de anastomoses e suturas realizadas com urgência sem a limpeza do intestino grosso, encorajando pesquisas científicas no sentido de suprimir essa etapa em cirurgias eletivas ${ }^{(5,6,7,8)}$.

A cicatrização das anastomoses realizadas no tubo digestivo representa preocupação constante devido a um risco relativamente alto de deiscência, o que resulta em alta morbidade e pode culminar na morte do paciente ${ }^{(9)}$.

Com este estudo pretendemos averiguar a influência do preparo intestinal sobre a anastomose colônica através da análise histomorfométrica, que consiste na observação do padrão inflamatório (agudo e crônico), presença de necrose e de calcificação, distribuição e quantidade das fibras colágenas. A reação inflamatória tem importância na cicatrização como demonstram diversos trabalhos, pois compreende uma seqüência de eventos que culminam em cicatrização ou cronificação do processo ${ }^{(9)}$. A necrose e a calcificação são complicações advindas desse processo inflamatório exacerbado, e têm relação com áreas de isquemia transitória decorrente de uma manipulação maior da parede intestinal. Já o colágeno é a peça principal da cicatrização, e sabe-se que uma maior quantidade e um maior grau de entrelaçamento de suas fibras são responsáveis por manter a força de tensão da anastomose $^{(10)}$.

\section{CASUÍTICA E MÉTODOS}

Foram utilizados 30 coelhos da raça Nova Zelândia com peso entre 2,0 e $3,0 \mathrm{~kg}$, submetidos a tratamento cirúrgico no Laboratório de Cirurgia experimental do Hospital Universitário da Universidade Federal de Sergipe, no período de agosto de 2004 a julho de 2005. Formaram-se dois grupos de forma aleatória, sem o conhecimento da equipe cirúrgica: grupo 1 (estudo), com 15 animais submetidos à ressecção e anastomose primária sem preparo do cólon, e grupo 2 (controle), com 15 animais submetidos à mesma cirurgia com LIATO.

Em todos os animais, realizou-se anestesia com associação das drogas: ketamina e cloridrato de xilazina. Posteriormente, foi realizada infiltração local com lidocaína a $1 \%$ com vasoconstrictor.

O primeiro tempo cirúrgico consistiu de abertura por planos e ressecção de um segmento do cólon a cinco centímetros da referência anatômica (terceiro giro do ceco) em ambos os grupos. No grupo 1, após ordenha manual das fezes, e no grupo 2, após a LIATO, era confeccionada a anastomose primária, plano único, total, com fio inabsorvível monofilamentar. O segundo tempo foi realizado no sétimo dia de pós- operatório, no qual foi realizada ressecção de um segmento da anastomose colônica para análise histomorfométrica.

Para cada segmento foram confeccionadas três lâminas histológicas, coradas pelas técnicas de hematoxilina-eosina (HE), picrosirius red e tricrômico de Masson. A avaliação histopatológica e histomorfométrica foi realizada através de microscopia óptica convencional e de luz polarizada. A análise do infiltrado inflamatório com quantificação diferencial das células bem como a avaliação de necrose e calcificação foi realizada nos cortes corados pela técnica de HE e a análise da fibrose, com quantificação e observação do grau de entrelaçamento das fibras do colágeno, foi realizada nos cortes corados por tricrômico de Masson e picrossirius red. A quantificação dos elementos celulares foi realizada por campos de média magnificação (100 aumentos) e do colágeno por campos de grande magnificação (400 aumentos).

Quanto à quantidade de colágeno, atribuiu-se nota zero (0) às anastomoses em que o colágeno foi desprezível; um (1) para presença de colágeno em pequenas quantidades; dois (2), em moderada quantidade e, três (3), quando encontrou-se grande quantidade de colágeno. No tocante à distribuição das fibras colágenas, observou-se dois padrões estruturais distintos: fibras em paralelo ou entrelaçadas. Considera-se que o entrelaçamento de fibras atribui uma maior força de tensão (resistência) no segmento em questão.

O infiltrado inflamatório foi avaliado de forma semi-quantitativa conforme os seguintes parâmetros: nota zero (0) para ausência do infiltrado inflamatório; um (1) para infiltrado leve; dois (2), moderado e três (3) pela presença de infiltrado inflamatório intenso. A necrose e a calcificação foram quantificadas em ter- 
Rev bras Coloproct Outubro/Dezembro, 2007
Estudo Histomorfométrico de Anastomoses Primárias de Cólon em Coelhos, Com e Sem Preparo Intestinal Juvenal da Rocha Torres Neto e Cols.
Vol. 27 mos percentuais em relação à área de infiltrado inflamatório peri-anastomose.

A análise estatística foi realizada com o auxílio do software Prism ${ }^{\circledR} 4$, com intervalo de confiança de $95 \%$, considerando que a $p$ é significativa se $<0,05$. Aplicou-se o teste de qui-quadrado para variáveis qualitativas (utilizando o teste de Fisher exato quando $>20 \%$ das casas da tabela $2 \times 2$ têm uma frequiência esperada $<5$ ), a prova " $t$ " de student para variáveis qualitativas com distribuição normal e a prova de Mann-Whitney para variáveis qualitativas cuja distribuição não é normal.

\section{RESULTADOS}

De acordo com o padrão estabelecido neste estudo, A presença de infiltrado inflamatório do tipo

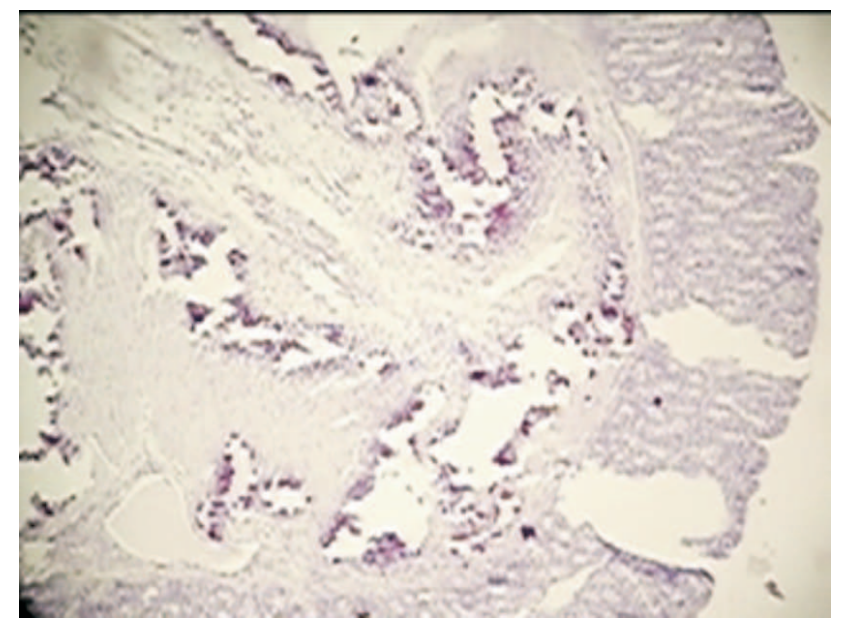

Figura 1 - Infiltrado inflamatório de moderada intensidade com lesão tecidual focal nas anastomoses com LIATO (HE, 100X).

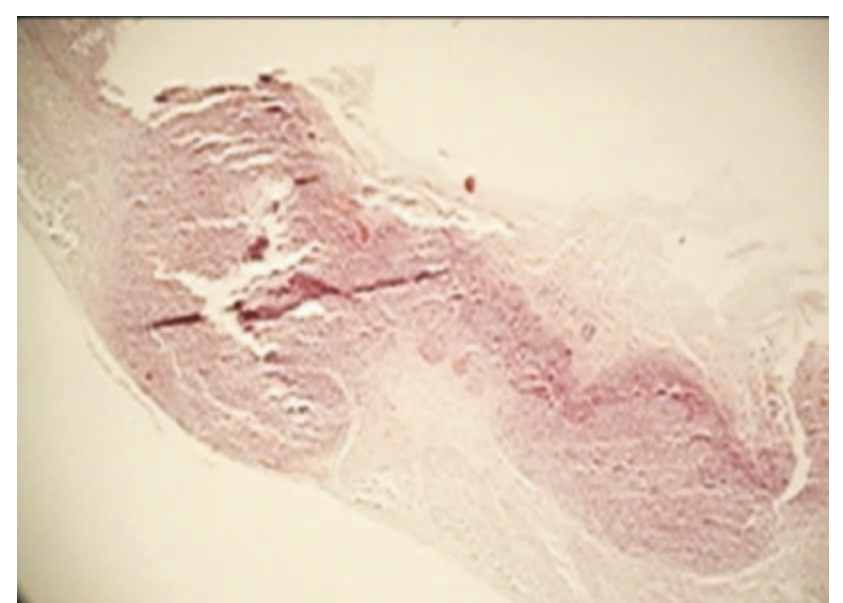

Figura 2 - Fibrose moderada a intensa peri-anastomose sem LIATO. Presença de foco de necrose no centro da anastomose (Tricromo de Masson, 40X). agudo (Figura-1) teve média de 1,6 nas anastomoses com LIATO. e de 1,4 nas sem preparo. Já a presença de infiltrado inflamatório do tipo crônico teve média de 1,9 nas anastomoses com LIATO e de 2,1 nas sem preparo. Para ambos os parâmentros o $\mathrm{P}$ foi maior que 0,05 (Gráfico 1).

A porcentagem de necrose (Figura. 2) nas cirurgias com LIATO foi de $15,7 \%$ contra $13,5 \%$ nas sem preparo. A presença de calcificações foi evidenciada em $43 \%$ das anastomoses com LIATO contra $30 \%$ nas sem preparo, com $\mathrm{P}>0,05$ (Gráfico 2).

Também com base nos valores padronizados em nosso estudo, observou-se uma maior concentração de colágeno em anastomoses feitas com LIATO, com uma média de 2,6. Nas anastomoses realizadas sem preparo a média para foi de 2,1 (Figura 3, Gráfico

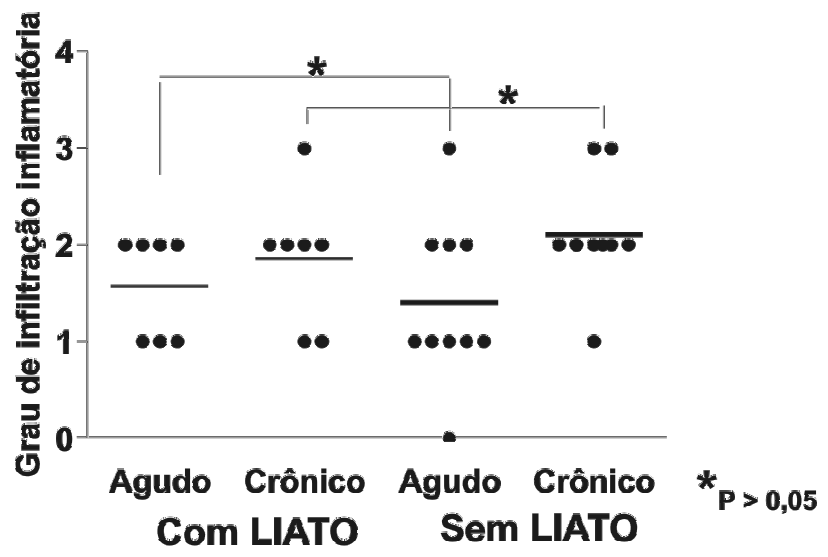

Gráfico 1 - Grau de infiltração inflamatória aguda e crônica, com e sem LIATO.

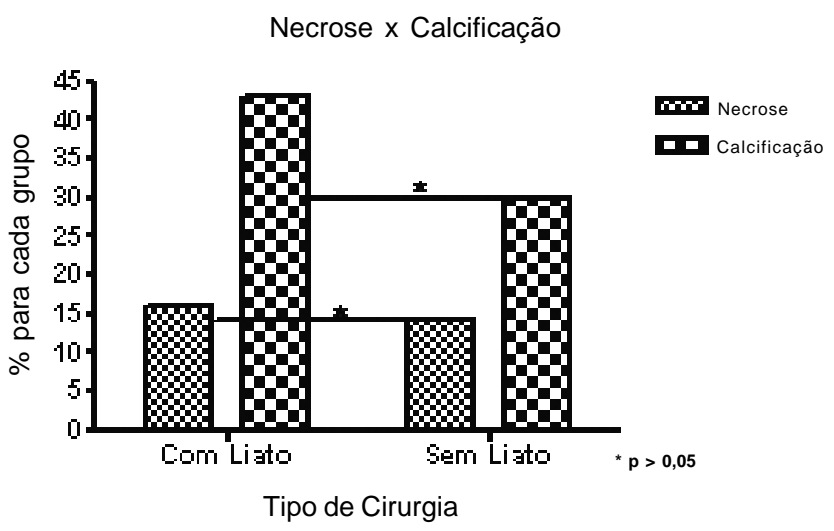

Gráfico 2 - Porcentagem de necrose e calcificação nas anastomoses com e sem LIATO. 
Rev bras Coloproct Outubro/Dezembro, 2007
Estudo Histomorfométrico de Anastomoses Primárias de Cólon em Coelhos, Com e Sem Preparo Intestinal Juvenal da Rocha Torres Neto e Cols.
3). A incidência de padrão entrelaçado foi vista em $86 \%$ das anastomoses do grupo com LIATO, enquanto que no outro grupo este percentual foi de 70\% (Figura.3, gráfico 4). Em nenhum dos parâmetros analisados houve diferença estatística significativa entre os dois gru$\operatorname{pos}(\mathrm{p}>0,05)$.

\section{DISCUSSÃO}

Vazamentos de anastomoses continuam a ser uma importante complicação na cirurgia colo-retal, resultando num aumento de morbidade e mortalidade. A despeito de melhoras nas técnicas cirúrgicas, existe ainda uma incidência de deiscência anastomótica superior a $15 \%$ em várias séries ${ }^{(11)}$.

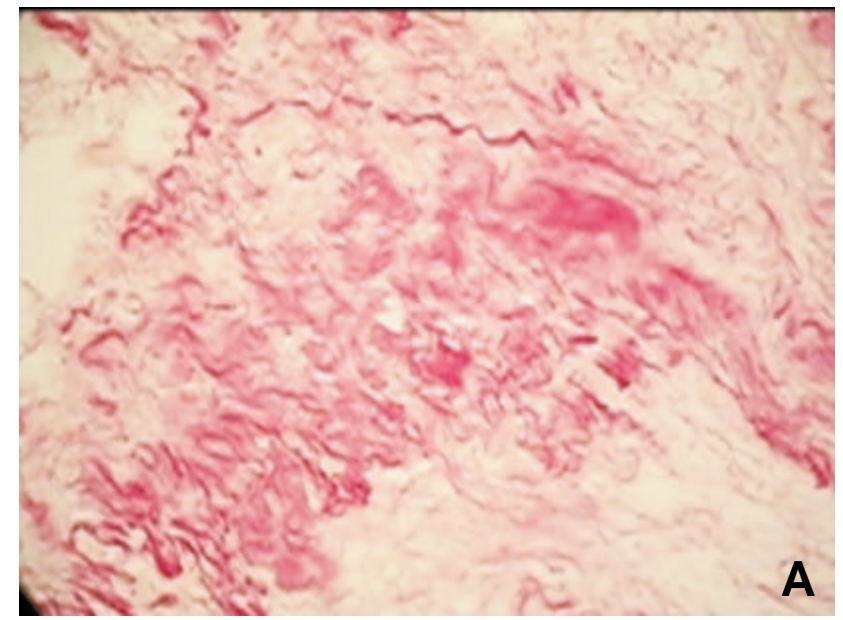

Existe discussão sobre como confeccionar uma anastomose: plano único ou dois planos, sutura contínua ou com pontos separados e o melhor fio a ser utilizado. Neste estudo optamos pela sutura contínua, em plano único, para a confecção das anastomoses colônicas. Este é um método simples, rápido e econômico, promovendo impermeabilidade e pouca reação inflamatória tecidual, sendo tão seguro quanto a sutura com pontos separados. Os fios monofilamentares são preferíveis aos multifilamentares, pois estes últimos favorecem o surgimento de infecções, acentuando a reação inflamatória ${ }^{(12,13)}$.

Estudos recentes vêm demonstrando o valor do conhecimento mais amplo e aprofundado do processo de cicatrização de feridas. Os parâmetros

Figura 3 - Fibras conjuntivas colágenas em anastomose com LIATO (A) mostrando média densidade e distribuição pouco entrelaçada e sem LIATO (B) mostrando grande densidade e distribuição acentuadamente entrelaçada (Picrossirus red, 400X).

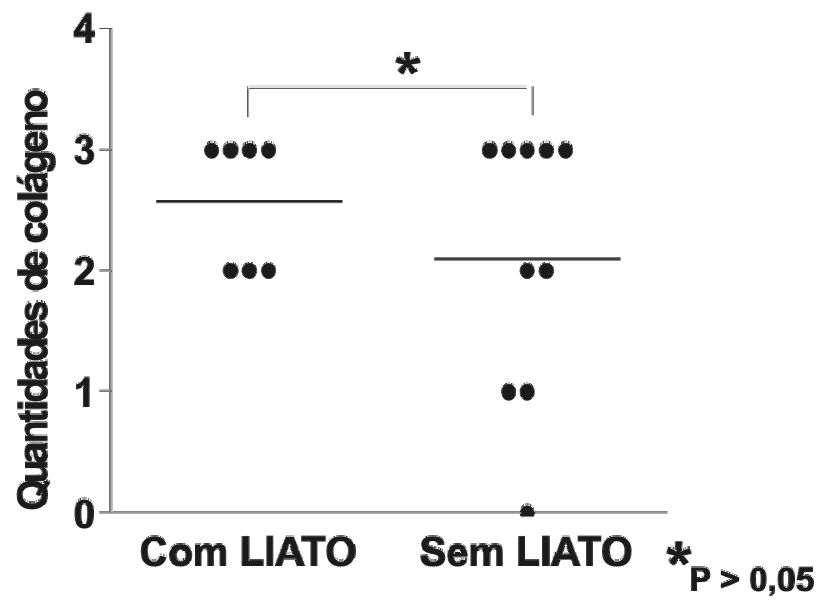

Gráfico 3 - Concentração de colágeno.

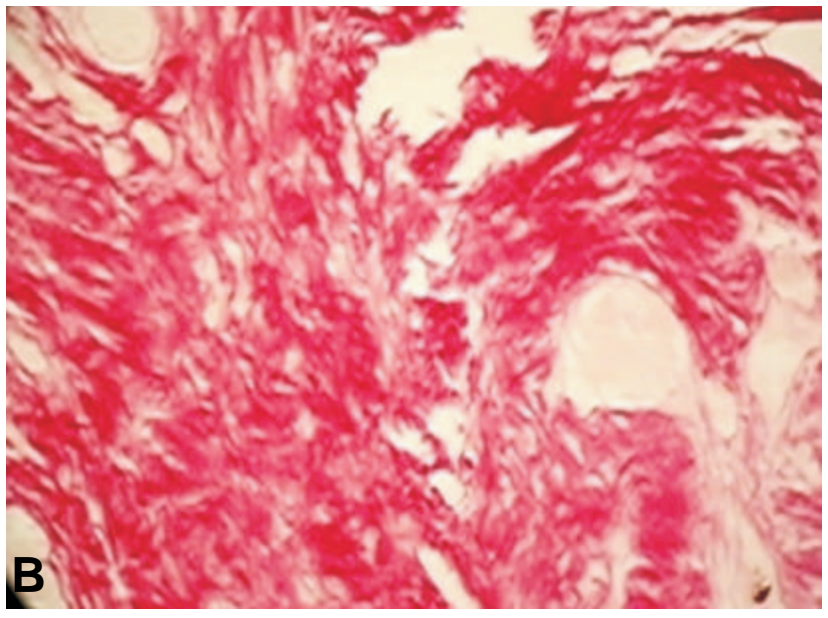

\section{(1)}


histológicos são muito úteis na descrição do processo e do resultado cicatricial. Eles foram escolhidos para avaliar os resultados deste estudo por serem precisos e facilmente quantificáveis. Através deles pode-se analisar a reação inflamatória tanto aguda como crônica, os graus de necrose e de calcificação, que estão relacionados com uma reação inflamatória exacerbada, e inferir a força da anastomose pela concentração e padrão de cruzamento das fibras colágenas ${ }^{(14)}$.

A fase inflamatória é a inicial da cicatrização, e é vital para o processo de reparação. Sem inflamação não há reparação ${ }^{(15)}$. A inflamação é fundamentalmente um mecanismo de defesa ${ }^{(10)}$ e de promoção da cicatrização ${ }^{(10)}$, entretanto, pode ser potencialmente prejudicial ao tecido, quando exacerbada. Ela leva a alterações importantes no processo cicatricial do intestino, resultando em cicatrizes com menor resistência e com menores quantidades de fibras colágenas quanto maior for o tempo e a intensidade da exposição do tecido aos mediadores inflamatórios ${ }^{(9,10)}$. Isso se deve ao fato de que a inflamação excessiva aumenta a atividade da colagenase, compromete a microcirculação e a proliferação de fibroblastos contribuindo para deiscência de anastomoses ${ }^{(9,16)}$.

Coradas pela técnica de hematoxilina-eosina, as lâminas deste estudo foram avaliadas por parâmetros histológicos clássicos; para a fase aguda e para a avançada. As anastomoses com LIATO apresentaram infiltrado inflamatório do tipo agudo mais incidente e infiltrado inflamatório do tipo crônico menos incidente, demonstrando que a limpeza do cólon diminuiu a resposta inflamatória exacerbada conferindo cicatrizes com menor sofrimento devido à menor agressão.

Complicações advindas do processo inflamatório exacerbado (notados pela maior incidência de calcificações e necrose) resultam em maior agressão tecidual. A necrose é a manifestação final de uma célula que sofreu lesões irreversíveis, caracterizando a morte celular ${ }^{17}$. Encontramos uma porcentagem maior de necrose e de calcificação no grupo submetido à LIATO apesar da reação inflamatória ter sido maior no grupo sem preparo. O maior tempo cirúrgico e a maior manipulação empregada no preparo intestinal intra-operatório podem ter provocado aumento da necrose nestes casos, já que a manipulação da parede intestinal leva a uma isquemia transitória que resulta em necrose e calcificação. No entanto, é necessário estudo subseqüente para maior avaliação destes parâmetros.
Sabe-se que a síntese do colágeno está diretamente ligada ao processo inflamatório. O colágeno é uma molécula vital em qualquer situação de reparo, sendo um dos fatores que mais contribuem na força e na integridade de todos tecidos agindo como base de sustentação dos mesmos. Distúrbios na matriz extracelular e da textura do colágeno desempenham papel importante na patogênese da deiscência anastomótica das cirurgias colo-retais ${ }^{(18)}$. No trato gastrintestinal, ele está localizado principalmente na submucosa ${ }^{(14)}$. Para que se mantenha a resistência mecânica da parede intestinal é necessário que ocorra um equilíbrio no processo de lise e de síntese do colágeno, fato este que ocorre na parede do intestino normal ${ }^{(14)}$. A proliferação fibroblástica e endotelial dá origem ao tecido de granulação. A síntese de colágeno pela ação dos fibroblastos, é mais intensa em torno do quinto ao sétimo dia de pós-operatório. A força de tensão da ferida aumenta com a formação de fibras colágenas e a adesão destas fibras entre si atinge o máximo entre o décimo quarto e o décimo sexto dia de pósoperatório ${ }^{(10)}$. Em função disso, realizamos a eutanásia dos coelhos no $7^{\circ}$ dia pós-operatório para a análise histomorfométrica, no ponto crítico da confecção das anastomoses intestinais. Apesar de após o $7^{\circ}$ dia aumentar a força tênsil da cicatriz optamos por não aguardar um maior tempo, por risco de morte por complicação dos animais de estudo. No nosso trabalho observamos que nas anastomoses realizadas com preparo havia maior concentração de colágeno que naquelas realizadas sem preparo, embora essa diferença não tenha sido estatisticamente significante demonstra uma tendência de as anastomoses com prévio preparo intestinal apresentarem melhor padrão de cicatrização.

A distribuição irregular (padrão de cruzamentos) das fibras colágenas aumenta o contato entre elas, promovendo maior resistência tênsil, conferindo maior segurança às anastomoses e conseqüientemente melhores resultados ${ }^{(19)}$. Existem estudos mais pormenorizados sobre o colágeno e a cicatrização das anastomoses, atribuindo-se uma maior importância ao colágeno tipo I e III para a qualidade tissular da parede intestinal levando a uma menor incidência de deiscência. No entanto, não realizamos tal diferenciação no presente estudo, o que poderemos realizar em futuros trabalhos. Utilizamos as colorações tricrômico de Masson e Picrosirius para análise da concentração e disposi- 
Rev bras Coloproct Outubro/Dezembro, 2007
Estudo Histomorfométrico de Anastomoses Primárias de Cólon em Coelhos, Com e Sem Preparo Intestinal Juvenal da Rocha Torres Neto e Cols. ção de colágeno, métodos específicos para detecção do colágeno ${ }^{(20)}$. A técnica do picrossirius red permite, além da quantificação do colágeno, analisar através da luz polarizada o padrão de disposição das fibras colágenas, avaliando seu arranjo arquitetural (entrelaçamento de fibras colágenas). Observamos que nas cirurgias realizadas com preparo, além da maior concentração de colágeno, havia um maior entrelaçamento de suas fibras. Novamente, demonstrando um melhor padrão cicatricial das anastomoses intestinais realizadas com preparo por maior adesão das fibras colágenas e, conseqüentemente maior resistência da sutura.

Apesar de os resultados deste estudo não terem apresentado significância estatística, eles sugerem que as anastomoses intestinais realizadas com preparo prévio apresentam maior segurança devido a menor infiltrado inflamatório crônico, maior concentração de colágeno e melhor padrão de entrelaçamento de suas fibras.

\section{CONCLUSÃO}

Através do estudo histomorfométrico das anastomoses intestinais com e sem preparo de cólon em coelhos, concluiu-se:

1. O infiltrado inflamatório do tipo agudo mais incidente e o infiltrado inflamatório do tipo crônico menos incidente nas anastomoses com LIATO demonstraram que a limpeza do cólon diminuiu a resposta inflamatória exacerbada tendendo a apresentar melhor padrão de cicatrização.

2. A presença de necrose e de calcificação pouco contribuiu para diferenças entre os grupos;

3. A realização da LIATO influencia positivamente no que diz respeito ao padrão de entrecruzamento das fibras colágenas, além de possibilitar maior concentração desta proteína nas áreas de reparo.

Sugere-se, dessa forma, que o preparo de cólon traz maior segurança ao se confeccionar anastomose intestinal.

ABSTRACT: Colon laudering is used in many colon surgeries. The LIATO, that promotes cleanness of colon, demonstrates an increase of the surgical time and increase risk of infection. This study compares colonic anastomosis with and without preparation, through histomorfometric analysis. 30 rabbits were submitted to the surgery treatment and had been evaluated and divided in groups: group 1 (control) and group 2 (LIATO). Carried through statistical study with the program Prism 4 for p<5\%. The analisis found acute inflammatory infiltrated with discrete bigger average in the anastomoses of group 2. Chronic inflammatory infiltrated with average of 1,9 in the anastomoses of group 2 and of 2,1 in the ones without preparation. Necrosis in $15,7 \%$ in the LIATO against $13,5 \%$ in the group without preparation. Calcium deposit in $43 \%$ of the anastomoses with preparation and in $30 \%$, of the ones without preparation. Bigger colágen concentration were met in the anastomoses made with the intestinal laudering. Incidence of interlaced colagen fibres was observed in $86 \%$ of the anastomoses of the searched one and in $70 \%$ in the control group. We conclude that the LIATO presents better incidence of interlaced colagen fibres and fibre concentration resulting in better anastomosis.

Key words: Anastomose, bowel, LIATO, healing, collagen.

\section{REFERÊNCIAS}

1. Moreira H, Moreira J P T, Moreira Júnior H - Limpeza do colo intra-operatório. Rev Bras Coloproct 2003; 23(3): p 187191

2. Stephenson BM, Shandall A A, Farouk R, Griffith G Malignant left-sided large bowel obstruction managed by subtotal/total colectomy. Br J Surg 1990; 77: p 1098-1102

3. Torralba J A, Robles R, Parrilla P, Lujan J A, Liron R, Pinero AEt Al - Subtotal colectomy $v$ s.intraoperative colonic irrigation in the management of obstructed left colon carcinoma. Dis Colon Rectum 1998; 41: p 18-22

4. Nascimento J E A, Caporossi C, Nascimento M - Comparação entre ressecção com anastomose primária e ressecção em estágios nos tumores obstrutivos do cólon esquerdo. Arq Gastroenterol 2002; 39(4): p 240-245

5. Azevedo J.L.M, Simões M.J, Stavale J.N- Estudo Comparativo entre as Anastomoses em plano único extramucoso e total de coelho. Acta Cir. Brasileira.1990.

6. Azevedo J.L.M, Simões M.J, Stavale J.N, Juliano N.F.N.Y. Avaliação da cicatrização e da integridade do colo anastomosado após rotação, em coelhos. Revista do Colégio Brasileiro de Cirurgiões, 1992; 19:3.

7. Massone.F. Anestesiologia Veterinária Farmacologia e Técnica. $4^{\mathrm{a}}$ edição. Ed. Guanabara Koogan, 2003. Cap. 3, pp 38-47.

8. Aronson,M. Slater Manual de Cirurgia de Pequenos Animais, Volume 1. $2^{\mathrm{a}}$ edição. Ed. Manole LTDA, 1993. Cap 45, pp. 751-759. 
9. Irvin TT, Golingher JC. Aetiology of disruption of intestinal anastomosis. Br J Surg; 1973;60:461-4 APUD: Mt, Bezuti et al . Cicatrização de anastomoses colônicas na vigência de obstrução intestinal: Estudo experimental em ratos. Acta Cir. Bras., São Paulo. 2002; 17:3:109-15

10. Kaplan, M.; Mentes, B.B.; Tatlicioglu, E.; Kayhan, B.; Aybay, C. Effect of mucosal immunomodulation with fed cholera toxin on healing of experimental colonic anastomosis. Dis. Colon Rectum. 2002;45:819-25. APUD: Goes Acam, Rodrigues Lv, Menezesdb, Grangeiro Mpf, Cavalcante Arms. Análise histológica da cicatrização da anastomose colônica, em ratos, sob ação de enema de Aroeira-do-sertão (Myracrodruon urundeuva fr. All.) A 10\%. Acta Cir Bras. 2005; Mar-Abr; 20(2).

11. Matthiessen P, Hallbook O, Rutegard J, Sjjodahl R Populationbased study of risk factors for postoperative death after anterior resection of the rectum. Br J Surg 2006; 93(4):498-503. APUD: Stumpf M., Krones C. J., Klinge U. Rosch R., Junge K., Schumpelick V. Collagen in colon disease. Hernia 2006; 10:498-501.

12. Skakun, G.B.; Reznick, R.K.; Bailey, H.R.; Smith, K.W.; Max, E. The single-layer continuous polypropylene colon anastomosis. A prospective assessment using watersoluble contrast enemas. Dis. Colon Rectum. 1988;31:163-8.

13. Katz, S.; Izhar, M.; Mirelman, D. Bacterial adherence to surgical sutures: a possible factor in suture induced infection. Ann. Surg. 1981;194:35-41.

14. Effects of Jatropha gossypiifolia L. (bellyache bush) extract on the healing process of colonic anastomosis: experimental study in rats. Acta Cir. Bras. 2006; 21:3:89-96

15. Marchini Fb, Martins Dmfs, Teves Dc, Simões Mj. Efeito do óleo de rosa mosqueta na cicatrização de feridas abertas. Rev. Paul. Med 1988; 106(6):356.

16. Mazzini DL, Mantovani M. Fechamento da parede abdominal com afastamento parcial das bordas da aponeurose utilizando sobreposição com telas de vicryil ou marlex em ratos. Acta Cir Bras. 1999; 14:1: 28-34. APUD: Brito Filho SB, Matias JEF, Stahlke Jr HJ, Torres OJM, Timi JRR, Tenório SB, Tâmbara EM, Carstens AG, Campos RV, Myamoto M. Análise da cicatrização na linha alba com uso de extrato aquoso de Orbignya phalerata (babaçu). Estudo controlado em ratos. Acta Cir Bras. 2006;21 Supl 3:76-88.

17. Kleiman I, Simões M J, Goldenberg S - Aspectos atuais do processo de reparação tecidual. Acta Cir Bras 1995; 10:2-8

18. Sakallioglu, AE.; Yagmurlu, A.; Dindar, H.; Hasirci, N.; Renda, N.; Deveci, MS. Sustained local application of low-dose epidermal growth factor on steroid-inhibited colonic wound healing. J. Pediatr. Surg. 1994;39:591-5. APUD: Goes ACAM, Rodrigues LV, Menezes DB, Grangeiro MPF, Cavalcante Arms. Análise histológica da cicatrização da anastomose colônica, em ratos, sob ação de enema de Aroeira-do-sertão (Myracrodruon urundeuva fr. All.) a 10\%. Acta Cir Bras. 2005 Mar-Abr; 20(2).

19. Tognini J R F, Goldenberg S, Simões M J, Sauer L, Melo R L, Ortiz PLA - Efeito do diclofenaco de sódio na cicatrização da parede abdominal de ratos. Acta Cir Bras 1998; 13(3): p 146154

20. Xavier R. - Oxytalan elastic and collagen fibers during the repair process in experimental nitric oxide inhibition. Clinics 60(2):85-92, 2005

\section{Endereço para correspondência:} JOSEANE SILVA SANTOS

Rua Silvio Romero, 302

Bairro Santo Antônio, Aracaju - SE 49060-630

Tel.: (79) 8107-9282 / 3215-3857

E-mail:joseane_ane@hotmail.com 